\title{
Ueber Verbindungen von Kohlehydraten mit
}

\section{Alkalien;}

von Dr. Th. Pfeiffer und B. Tollens.

(Eingelaufen am 6. September 1881.)

In vielen Uebersichten der Kohlehydrate findet man für die folgenden Gruppen die nebenstehenden Formeln :

Gruppe der Glycosen, $\quad \mathrm{C}_{6} \mathrm{H}_{12} \mathrm{O}_{6}$,

》 des Rohrzuckers, $\mathrm{C}_{12} \mathrm{H}_{24} \mathrm{O}_{14}$,

ᄁ der Stärke, $\quad \mathrm{C}_{6} \mathrm{H}_{10} \mathrm{O}_{5}$ oder $\left(\mathrm{C}_{6} \mathrm{H}_{10} \mathrm{O}_{5}\right)_{6}$.

Wenn nun für die beiden ersten Gruppen die obige Formel und Moleculargröfse wohl nach ihrem ganzen Verhalten festgestellt ist, so ist dieses für die Glieder der Stärkegruppe, d. h. die Stärke selbst, die Dextrin- und Gummiarten, das Inulin u. s. w. keineswegs der Fall, vielmehr sind Angaben mehrfacher Art vorhanden, nach welchen einerseits die Moleculargröfse eine sehr viel bedeutendere sein muls, andererseits auch die durch die Formel $\mathrm{C}_{6} \mathrm{H}_{10} \mathrm{O}_{5}$ ausgedrückte procentische Zusammensetzung nicht mit den Resultaten der Analyse genau stimmt.

So giebt in Folge kleiner Differenzen, welche die Resultate der Analyse mit den für obige Formel geforderten Mengen zeigen, W. Nägeli *) als Zusammensetzung der Stärke die Formel $\mathrm{C}_{36} \mathrm{H}_{62} \mathrm{O}_{31}$, welche sich von dem sechsfachen der obigen einfachen Formel oder $\mathrm{C}_{36} \mathrm{H}_{60} \mathrm{O}_{90}$ durch Mehrgehalt von $\mathrm{H}_{2} \mathrm{O}$ unterscheidet. R. S a chs $\mathrm{e}^{* *}$ ) schliefst sich dieser Ansicht unter Zugrundelegung der bei der Ueberführung von

*) Dr. W. Nageli, Beitrăge zur năheren Kenntnifs der Stărkegruppe, Leipzig 1874, S. 33, 99.

*) Chemisches Centralblatt [8] 8, 732 . 
Stärke in Dextrose auftretenden Zahlenverhältnisse an, welche wohl mit der Gleichung $\mathrm{C}_{85} \mathrm{H}_{6 z} \mathrm{O}_{31}+5 \mathrm{H}_{8} \mathrm{O}=6 \mathrm{C}_{6} \mathrm{H}_{12} \mathrm{O}_{6}$, aber nicht mit $\mathrm{C}_{6} \mathrm{H}_{10} \mathrm{O}_{5}+\mathrm{H}_{9} \mathrm{O}=\mathrm{C}_{6} \mathrm{H}_{18} \mathrm{O}_{6}$ stimmen. Eine gleiche Zusammensetzung schreibt weiter $\mathrm{Nägeli*}$ ) dem von ihm untersuehten Amylodextrin, sowie auch dem Dextrin zu.

Nach den bei Untersuchung der Verzuckerung der Stärke dorch Malz gewonnenen Resultaten wird ferner der Stärke von manchen Autoren ein gröfseres und z. Th. ein sehr grofses Moleculargewicht beigelcgt. Freilich wird nicht immer klar ausgesprochen, ob die Anzahl von Atomen, welche als Stärke in Reaction treten und Dextrin und Maltose bilden, vorher zu einem Molecul verbunden waren, oder ob etwa mehrere kleinere Molecule zusammen in Action treten.

So lassen O'Sullivan **), Märker **) und $E$. $\mathrm{Schulze} f$ ) drei resp. vier Gruppen $\mathrm{C}_{6} \mathrm{H}_{10} \mathrm{O}_{5}$ an der Zersetzung Theil nehmen, und geben Brown und Heron + ) Reactionsgleichungen, in denen zehn Gruppen $\mathrm{C}_{\mathbf{1 y}} \mathrm{H}_{\mathbf{2 0}} \mathrm{O}_{\mathbf{1 0}}$ figuriren. O'S ulliv a n scheint der Ansicht zu sein, dars die einzelnen Stärkemolecule 18 Atome Kohlenstoff enthalten, und Brown und Heron weisen auf die Ansicht von Armstrong hin, wonach 72 oder gar 144 At. Kohlenstoff in einem Molecul Stärke sich befinden. Mus culus und Gruber $+f+$ ) endich sehen die Stärke als ein Polysaccharid von der Formel $n\left(\mathrm{C}_{18} \mathrm{H}_{20} \mathrm{O}_{10}\right)$ an, worin $\mathrm{n}$ nicht unter 5 oder 6.

Zu einem Urtheile über die Gröfse des Stärkemoleculs

*) Nuge li, Starkegruppe, S. 103.

*) Journ. of the Chemical Soc. 14, 141.

*) Landwirthschaftliche Jahrbïcher 1, 313.

†) Journ. fir Lendwirthsch. 26, 72.

14) Diese Annalen 189, 242.

Hi) Bulletin de la Société [2] $30,69$. 
mufste man kommen, wenn es gelang, in dasselbe, ähnlich we es bei Bestimmang der Moleculargrörse von Săuren geschebt, ein Atom eines Metalles einzuführen, wozu sich natürlich am besten einwerthige Metalle eignen.

Derartige Verbindungen der Stärke mit z. B. Silber, Kalium, Natrium sind früher nicht rein hergestellt worden.

Dagegen sind von anderen Kohlehydraten einige derartige Verbindungen bekannt; so haben Brendecke ${ }^{*}$ ) und So ub oi ra ${ }^{* *}$ ) die Alkaliverbindungen des Rohrzuckers, B rendecke diejenigen des Stärke- und des Milchzuckers, Hönig und Rosenfeld**) die Natriumverbindungen der Dextrose, Lävulose und des Milchzuckers, Herzfeld $f$ ) diejenige der Maltose hergestellt, und es hat sich hier, wenigstens nach den neueren Untersuchungen, ergeben, dafs in der That in den Natriumderivaten der Maltose, des Milchzuckers und des Rohrzuckers auf 1 At. Natrium 12 At. Kohlenstoff, in den Natriumderivaten der Glycosen dagegen nur 6 At. Kohlenstoff auf 1 At. Natrium vorhanden sind. Complicirtere Resultate haben die verschiedentlich hergestellten Derivate mit Calcium, Baryum nnd Blei, sowie die Versuche Drag en dorff's mit Inulinnatrium und -kalium $+t$ ) ergeben.

Der Eine von uns hat schon vor längerer Zeit $+f+$ ) Natrium- und Kaliumderivate der Stärke in reinem Zustande zu gewinnen versucht, und die Resultate der verschiedenen Versuche zeigten, dafs in diesen Körpern auf 1 At. Natrium 24 bis 30 At. Kohlenstoff vorhanden sind.

*) Archir d. Pharmacio [2] $8 \mathbf{4}, 71$.

*) Diese Annalen 48, 223.

**) Berichte der deutschen chem. Gesellschaft 10, 871; $19,45$.

†) Herafeld, Dissertation, Halle 1879, S. 12.

H) Dragend orff, Materialien zu einer Monographio des Inulins, Petersburg 1870, S. 121.

tit) Joumal f. Landwirthschaft 1873, S. 375. 
Wir haben diese Versuche fortgesetzt und aufser der Stărke auch Amylodextrin, Inulin und Dextrin in den Kreis der Untersuchungen hereingezogén.

\section{Stärke.}

\section{A. Natriumverbindung.}

Wenn man Stärke mit kaltem Wasser anrührt und Natronlauge hinzufügt, erhält man eine kauın gelbliche, durchscheinende Gallerte. Alkohol scheidet aus der völlig homogenen Masse, wie früher schon Bé cham p*) gezeigt, einen anfangs sehr zähen, schwer zu bearbeitenden Körper ab, der jedoch bei wiederholtem Verreiben mit Alkohol resp. schliefslich Aether allmälig körnig und pulverig wird, so dafs man ihn nach dem Trocknen über Schwefelsäure als eine weifse, äufserlich der Stärke sehr ähnliche Substanz gewinnt. Dieselbe ist unter dem Mikroskop völlig amorph, sie reagirt stark alkalisch und unterscheidet sich ferner durch die Löslichkeit in kaltem Wasser von der Stärke. Mit Jod und etwas Săure zeigt sie dagegen sofort die charakteristische Blaufärbung der Stärke. Weder durch Ausfrieren noch durch langsames Verdunsten der Lösung über Schwefelsäure konnte die Verbindung irgendwie krystallinisch erhalten werden.

Das Natrium ist sehr lose gebunden und trennt sich mit Wasser schon theilweise als Natriumhydroxyd, mit Säuren vollständig als Natriumsalz ab, so dafs man das Natrium durch Titrirung mit verdünnter Schwefelsäure auch ohne Veraschung der Substanz bestimmen kann und es ungewifs ist, ob eine Zusammenlagerung von Stärke mit $\mathrm{NaOH}$ oder ein Eintritt von Natrium in die Stärke wahrscheinlicher ist.

Wir haben käufliche, vorher noch mit Wasser ausge-

*) Journal de Pharmacie [3] D 2, 409. 
waschene Kartoffelstãrke verwandt. Sie verlor beim Trocknen bei $105^{\circ} 15,25$ und $15,18 \mathrm{pC}$. an Wasser und liefs an Asche 0,47 und $0,39 \mathrm{pC}$.

Das Natrium haben wir in Gestalt von Natriumalkoholat eingeführt und zwar stets die erforderliche Menge einer Lösung von $1 \mathrm{~g}$ Natrium in $20 \mathrm{ccm}$ Alkohol, d. h. so wenig Alkohol, dafs noch keine Fällung erfolgen konnte.

Die abgewogene Stärke wurde mit abgemessenen Mengen kalten Wassers in einem Porcellanmörser gut verrieben und darauf das Natriumalkoholat allmälig hinzugefügt.

Sobald die Masse eine homogene Gallerte war, das Natriumalkoholat also auf alle Theile gleichmäfsig eingewirkt hatte, wurde die Fällung mit 95 procentigem Alkohol vorgenommen. Unter häufigem Abgiefsen der überstehenden Flüssigkeit, Hinzubringen von neuem 95procentigem oder zuweilen absolutem Alkohol und geduldigem Verarbeiten des sehr zähen, allmälig härter, körnig, zuletzt pulverig werdenden Teiges, erhält man schliefslich die bereits kurz charakterisirte Verbindung.

Zahlreiche Versuche sind mit verschiedenen Mengen Natrium und Wasser ausgeführt und anderen Orts *) im einzelnen reproducirt.

Bei diesen Versuchen variiren die gefundenen Natriumprocente von 1,32 bis $7,07 \mathrm{pC}$., und es hat sich herausgestellt, dafs die Quantität des ursprünglich zugesetzten Natriums auf den Procentgehalt des entstehenden Products von nicht sehr grofser Bedeutung ist, z. B. :

$\begin{array}{ccc}\text { Stärke } & \text { Natrium } & \begin{array}{c}\text { Gehalt des Productes } \\ \text { an Natrium **) }\end{array} \\ 5 \mathrm{~g} & 0,5 \mathrm{~g} & 4,50 \mathrm{pC} . \\ 5 \mathrm{~g} & 1,0 \mathrm{~g} & 4,99 \mathrm{pC} . \\ 5 \mathrm{~g} & 1,5 \mathrm{~g} & 4,72 \mathrm{pC} .\end{array}$

-) Pfeiffer, Inauguraldissertation, Göttingen 1881, S. 11 bis 12.

*) Stets Durchschnitt von drei gut stimmenden Analysen, z. B. 4,56 pC., 4,49 pC., 4,45 pC. Die über Schwefelskure getrocknete 
Von sehr grofsem Einflufs ist dagegen die Art der Darstellung, besonders die Reinigung. Wäscht man das Pulver auf dem Filter mit Alkohol und Aether aus, so ist es kaum möglich, die alkalische Reaction der Waschflüssigkeit genügend herabzubringen, um sie vernachlässigen zu können, es bleibt somit leicht eine nicht unbecieutende Menge von Natriumalkoholat in der auf dem Filter sich befindenden Substanz. So ergaben die ersten Versuche einen Gehalt von $5,48,5,80$, $6,98 \mathrm{pC}$. Natrium.

Geringere Zahlen erhält man, wenn man durch mehrmaliges Anreiben mit neuem Alkohol und Decantiren die Hauptmenge des Natrons fortschafft und erst nachträglich wäscht, z. B. die oben angeführten Zahlen; besonders aber erhält man geringere Merigen Natron, wenn man das mit Alkohol gefällte, pulverig gewordene Product nach dem $\mathbf{A b}-$ giefsen des Alkohols in möglichst wenig Wasser löst und mit Alkohol wieder fällt, und stets geringere Zahlen, wenn man diefs Verfahren wiederholt.

Wir haben diefs genau constatirt, indem wir stets je 5 (resp. 10) g Stärke mit $50 \mathrm{ccm}$ Wasser und $0,5 \mathrm{~g}$ Natrium als Alkoholat (resp. $100 \mathrm{ccm}$ und $1 \mathrm{~g}$ ) in Arbeit nahmen und die verschiedenen Präparate resp. einmal, zweimal bis sechsmal wieder lösten und fällten. Wir erhielten so, während bei einmaliger Fällung die obigen Zahlen 4,50 bis 4,99 pC. resultirten, bei doppelter Fällung 3,55 pC. Natrium,

$$
\text { , dreifacher }, 2,55 \text {, }
$$

Substanz wurde vor der Analyse bei 96 bis $98^{\circ}$ bis zu constantem Gewicht getrocknet und orlitt hierbei einen Verlust von 6,75 bis $10,56 \mathrm{pC}$. Der Natriumgehalt wurde durch Lösen in titrirter Bchwefelsüure und Zurücktitriren mit Barytwaseer unter Anwendung von Rosolskure ermittelt. Hierbei wurde, um $\mathrm{Zu}$ sammenballen zn verhindern, die Natriumverbindnng vorher mit Alkohol befouchtet. 
bei vierfacher Fällung 2,00 pC. Natrium,

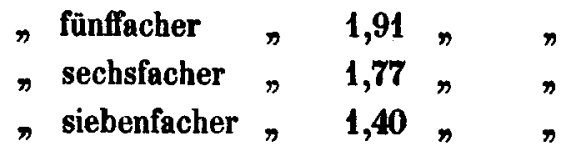

Es war hierdurch klar, dafs bei wiederholter Fällung Zersetzung unter Verlust von Natrium eintritt, andererseits ist ebenso klar, dafs bei nur einmaligem Fällen von solchen amorphen gallertartigen, allmälig festwerdenden Stoffen mehr oder weniger von der Mutterlauge eingeschlossen bleibt, oder auch durch Oberflächenattraction u. s. w. dem Niederschlage antraftet *); denn wie bei der Bereitung des Dextrins mehrmalige Fällung unerläfslich ist, um es einigermafsen zuckerfrei zu bekommen, so mufs man sogar manche Niederschläge der unorganischen Analyse mehrmals fällen, um sie rein zu erhalten.

Wird nun die Substanz noch einmal in Wasser gelột, so vertheilt sich auch der Alkaliüberschuifs in einer gröfseren Fhüssigkeitsmenge, und nur ein ganz geringer Procentsatz wird wieder beim Fällen mechanisch zurückgehalten, so dafs hiernach die Verbindung wohl als rein angesehen werden kann. Bei dieser zweiten Fällung kann allerdings auch schon eine geringe Zersetzung stattgefunden haben und hierdurch der Natriumgehalt verringert sein, und schwer ist es, die beiden Klippen, nämlich das Niederschlagen von zuviel Alkali boi zu geringer Zahl von Fällungen und die Zersetzung der Substanz unter Verlust von Alkali bei zu häufiger Fällung zu vermeiden. Wir haben uns dahin entschieden, die zweimal gefältten Niederschläge als diejenigen $\mathrm{zu}$ betrachten, bei welchen die genannten Fehlerquellen am wenigsten vorhanden sind oder sich

*) Ob diefs Anhaften auf Flachemattraction u. s. W., oder anf der Existenz einer natriumreicheren losen Verbindung beroht, ist schwer so entscheiden is. a. van Bommelen, Journal futir praktischo Chemie [2] $\mathbf{2 8}, 392)$. 
am besten compensiren, weil wir nur bei zweimaliger Fällung wirklich constante Zahlen erhalten haben, und somit haben wir bei allen Kohlehydraten diefs Verfahren eingeschlagen. Wie sich hieraus natürlich ergiebt, können wir die gefundenen Formeln nicht ganz genau als Ausdruck der Moleculargrö[se hinstellen, da wir aber bei Herstellung der verschiedenen Verbindungen stets dasselbe Verfahren beobachtet haben, so gestatten die Zahlen jedenfalls einen Vergleich zwischen den Moleculargröfsen verschiedener Kohlehydrate.

Eine andere Fehlerquelle bringt die kaum genügend auszuschliefsende Kohlensäure der Luft, denn sie zersetzt die Verbindung und es bleiben, wenn letztere dann wieder gelöst und gefällt wird, die doch immerhin nur geringen entstandenen Mengen Natriumcarbonat theilweise in Lösung, so dafs der Natriumgehalt geringer wird; das in der Substanz vorhandene Natrium ist aber dann ebenfalls $z$. Th. als Carbonat darin, und in der That haben wir gefunden, dafs ein erheblicher Gehalt an Kohlensäure mit geringen Natriumprocenten Hand in Hand geht. So lieferte einmal ein doppelt gefälltes Product neben 2,53 und 2,49 pC. $\mathrm{CO}_{2}$ nur 2,78 und 2,86 pC. $\mathrm{Na}$, und das Product der sogleich folgenden Bereitung I mit 3,49 und 3,44 pC. Na gab, als es ohne Vorsichtsmafsregeln einige Stunden im Trockenschrank verweilt hatte, beim Wiederlösen und Fällen eine Verbindung mit nur 1,89 pC. Na.

Dagegen gaben neue, unter möglichstem Ausschluls von Kohlensäure bereitete (indem rasch gearbeitet wurde, und in dem Trockenschrank sich stets Gefälse mit Kalk befanden) Portionen folgende Zahlen :

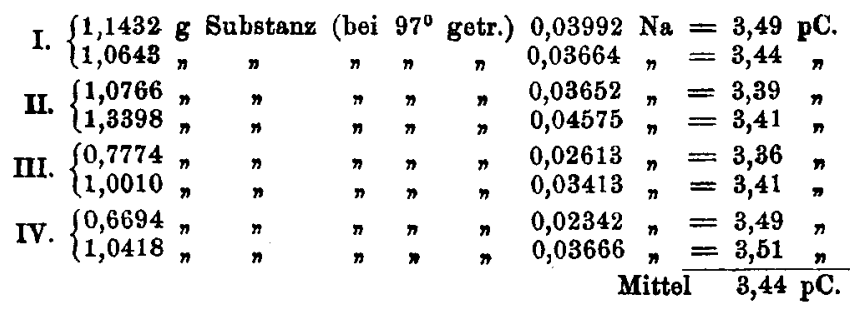


Von dem Gemenge der Bereitungen II bis IV gaben :

2,5881 g $0,0138 \mathrm{CO}_{\mathbf{8}}=0,55 \mathrm{pC}$,

2,1975 g $0,0123 \mathrm{CO}_{2}=0,56 \mathrm{pC}$.,

ferner gab die bei $105^{\circ}$ getrocknete Substanz bei der Verbrennung folgende Daten ${ }^{*}$ :

I. $0,4481 \mathrm{~g}$ gaben $0,6920 \mathrm{CO}_{2}$ und $0,2351 \mathrm{H}_{8} \mathrm{O}$.

II. $0,3624 \mathrm{~g}$, 0,5574 , 0,1914 ,

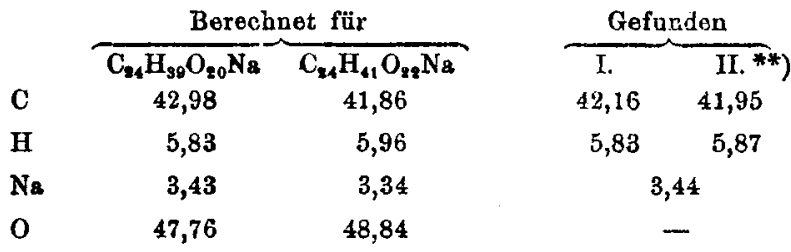

Man erhält also, wenn man genau wie oben angegeben arbeitet, eine Verbindung, in welcher 24 At. Kohlenstoff auf 1 At. Natrium vorhanden sind.

\section{B. Stärkekalium.}

Die entsprechenden Kaliumverbindungen haben wir unter möglichst gleichmäfsigen Bedingungen wie die Natriumverbindung und stets durch doppelte Fällung dargestellt.

Wir haben reinstes käufliches Kaliumhydroxyd in Alkohol gelöst, von einem geringen Bodensatz decantirt und wie

*) Die Verbrennungen wurden mit einem leicht schmelzbaren Gemenge yon Blei- und Kaliamchromat und vorgelegtem Kupferoxyd susgeführt.

*) Zur Natriumbestimmung ist die Substanz bei $97^{\circ}$ (im Wassertrockenschrank), zur Verbrennung bei $105^{\circ}$ getrocknet worden. Wio ein Versuch zeigte, verliert sio zwischen 97 und $105^{\circ}$ noch 2,77 pC. an Gewicht, doch ist diefs in Bezug auf den Natrinmgehalt ohno Belang. Eben so wenig Rucksicht haben wir auf den Gehalt der Substanz von 0,55 pC. Kohlensäure genommen, weil das Resultat nicht geändert wird, wenn man 0,55 pC. $\mathrm{CO}_{2}$ von der Substanz und die entsprechende Menge Kohlenstoff oder 0,15 pC. von dem erbaltenen Kohlenstoff abzieht. 
bisher das Doppelte der für die erwartete Verbindung berechneten Menge, also 1,8 $\mathrm{g} \mathrm{KOH}$ auf $10 \mathrm{~g}$ Stärke angewandt.

Bei der Fällung mit Alkohol erhält man eine etwas lockerere Masse als bei der Natriumverbindung, die sich besser und rascher verarbeiten läfst. Sie ist ferner in kaltem Wasser etwas leichter löslich als die Natriumverbindung.

Kaliumbestimmungen :

I. a. $0,6689 \mathrm{~g}$ Substanz gaben $0,03503 \mathrm{~K}=5,24 \mathrm{pC}$.

b. $0,9723 \mathrm{~g} n$ n $0,04991 \mathrm{~K}=5,13 \mathrm{pC}$.

II. a. $0,6172 \mathrm{~g} n, \quad n \quad 0,03289 \mathrm{~K}=5,33 \mathrm{pC}$.

b. $1,0869 \mathrm{~g} n$ n $0,05794 \mathrm{~K}=5,33$ pC.

III. a. $0,7474 \mathrm{~g} n \quad n \quad 0,03948 \mathrm{~K}=528 \mathrm{pC}$.

b. $0,8200 \mathrm{~g} n \rightarrow 0,04273 \mathrm{~K}=5,21 \mathrm{pC}$.

Durchschnitt $=5,25 \mathrm{pC}$

Kohlensäurebsutimmung :

$2,6650 \mathrm{~g}$ Substanz gaben $0,0485 \mathrm{CO}_{\mathrm{z}}=1,82 \mathrm{pC}$.

$2,3010 \mathrm{~g}$ " $0,0420 \mathrm{CO}_{2}=1,82 \mathrm{pC}$.

Trotz aller Vorsichtsmafsregeln war also ziemlich viel Kaliumcarbonat gebildel worden, das wohl zum gröfsten Theil in der präcipitirten Substanz geblieben ist. Etwas wird aber auch aller Wahrscheinlichkeit nach ausgewaschen sein.

\begin{tabular}{|c|c|c|c|}
\hline & Berechnet für & & Gefunden \\
\hline$\widetilde{\mathrm{C}}_{94} \mathrm{H}_{98} \mathrm{O}_{90} \mathrm{~K}$ & $\mathrm{C}_{24} \mathrm{H}_{41} \mathrm{O}_{21} \mathrm{~K}$ & $\mathrm{C}_{80} \mathrm{H}_{49} \mathrm{O}_{25} \mathrm{~K}$ & Mittel \\
\hline 5,69 & 5,54 & 4,59 & 5,25 \\
\hline
\end{tabular}

Der gefundene Gehalt an Kalium liegt also in der Mitte der von Formeln mit resp. 24 und 30 Atomen Kohlenstoff geforderten Mengen, jedoch näher an der Formel mit 24 Atomen Kohlenstoff, welche der aus der Stärkenatriumverbindung berechneten analog ist, und am nächsten einer solchen, welche noch $\mathrm{H}_{8} \mathrm{O}$ enthält und sich in $\mathrm{C}_{24} \mathrm{H}_{40} \mathrm{O}_{20}+\mathrm{KOH}$ zerlegen lärst.

Die Formel der Stärke, welche diesen Natrium- und Kaliumderivaten entspricht, ist $\mathrm{C}_{24} \mathrm{H}_{4,} \mathrm{O}_{20}$ oder $\mathrm{C}_{24} \mathrm{H}_{42} \mathrm{O}_{21}$ 
$\left(=\mathrm{C}_{24} \mathrm{H}_{40} \mathrm{O}_{20}+\mathrm{H}_{2} \mathrm{O}\right)$ und enthält also viermal die Gruppe $\mathrm{C}_{6} \mathrm{H}_{10} \mathrm{O}_{5}^{*}$ ).

Sie weicht von der Formel von $\mathrm{Nägeli}$ und S achsse $\mathrm{ab}$, welche sechsmal die Gruppe $\mathrm{C}_{6} \mathrm{H}_{10} \mathrm{O}_{5}$ enthält, und wir enthalten uns jedes Urtheils darüber, $o b$ die von uns erhaltenen Andeutungen über die Moleculargröfse der Stärke oder diejenigen von $\mathrm{Nägeli}$ und $\mathrm{Sachss}$ e die mafsgebenden sind, denn, wie oben gesagt, ist bei unseren Darstellungen stets die Ungewifsheit vorhanden, ob wir zwischen den genannten Klippen des Zuviel- oder Zuwenigerhaltens von Natrium resp. Kalium die richtige Mitte eingehalten haben.

Wenn man annimmt, dafs in unseren Versuchen auch bei der zweiten Fällung mechanisch Natrium resp. Kalium niedergerissen seien, was uns jedoch sehr unwahrscheinlich scheint, kommt man auf Formeln mit mehr Kohlenstoff, wenn man dagegen schon bei der zweiten Fällung erhebliche Zersetzung annimmt, kommt man auf Formeln mit weniger Kohlenstoff, z. B. mit nur dreimal der gewöhnlichen Stärkegruppe. Wenn nicht das Resultat der zweimaligen Fällung, sondern dasjenige der dreimaligen das mafsgebende ist, kommt man den nach $\mathrm{C}_{36} \mathrm{H}_{59} \mathrm{NaO}_{30}$ oder $\mathrm{C}_{36} \mathrm{H}_{61} \mathrm{NaO}_{i 1}$ berechneten Procentgebalten resp. oder 2,31 und $2,27 \mathrm{pC}$. Natrium sehr nahe, denn wie oben angegeben sind von uns bei dreimaliger Fällung 2,55 pC., bei viermaliger Fällung 2,00 pC. gefunden.

Anderereits möchten auch die von W. $\mathrm{Näg}$ eli erhaltenen, auf $\mathrm{C}_{96} \mathrm{H}_{62} \mathrm{O}_{31}$ passenden Zahlen der Stärkeanalysen, wenn auch weniger doch annähernd auf die Formel $\mathrm{C}_{24} \mathrm{H}_{42} \mathrm{O}_{21}$ pas-

*) Es versteht sich ron selbst, dafs das Urtheil nur die Stärke, wie wir sie vorwandten, betrifft, Stärkekleister haben wir in dieser Hinsicht nicht untersucht. 
seri, und dasselbe möchte für die $\mathrm{S}$ a chs se'schen, auf die quantitative Bestimmung der aus Stärke zu gewinnenden Menge Dextrose gebauten Schlüsse gelten, wenigstens stimmt die nach der Gleichung :

$$
\mathrm{C}_{24} \mathrm{H}_{42} \mathrm{O}_{21}+3 \mathrm{H}_{2} \mathrm{O}=4 \mathrm{C}_{6} \mathrm{H}_{12} \mathrm{O}_{6}
$$

zu erhaltende Menge Dextrose $(108,11$ pC.) besser mit der nach :

$$
\mathrm{C}_{36} \mathrm{H}_{62} \mathrm{O}_{31}+5 \mathrm{H}_{2} \mathrm{O}=6 \mathrm{C}_{6} \mathrm{H}_{12} \mathrm{O}_{6} \text {, }
$$

sich ergebenden Quantität $(109,09 \mathrm{pC}$.), als mit den nach der alten Gleichung :

$$
\mathrm{C}_{6} \mathrm{H}_{10} \mathrm{O}_{5}+\mathrm{H}_{2} \mathrm{O}
$$
resultirenden Zahlen (111,11 pC.).

\section{Rohrzucker.}

\section{A. Rohrzuckernatrium.}

Je $5 \mathrm{~g}$ Rohrzucker wurden in $25 \mathrm{ccm}$ Wasser gelöst, mil $0,7 \mathrm{~g}$ Natrium als Alkoholat versetzt und mit Alkohol gefällt, und zwar zwei-, drei-, fünf- und siebenmal.

Das erhaltene Product schwoll, wie sich bei der Analyse der zweimal gefällten Probe zeigte, bei $97^{\circ}$ stark auf, bräunte und zersetzte sich etwas und die niedrige Procentzahl dieser Portion (I.) an Natrium mag zum Theil hierauf beruhen (siehe unten).

Deshalb wurde bei den drei-, fünf-, siebenmal gefallten Proben die nur uber Schwefelsuure getrocknete Substanz titrirt und nach dem Verlust einer gleichzeitig bei $97^{\circ}$ getrookneten Probe das Gewicht berechnet, welches die zum Titriren angewand te Probe bei $97^{\circ}$ angenommen haben würde. Diese Verluste sind übrigens nur gering gewesen, denn II. hat 0,64 pC., III. 2,52 pC. und IV. gar nicht abgenommen. 


\begin{tabular}{|c|c|c|c|c|c|}
\hline $\begin{array}{l}\text { Nr. des } \\
\text { Yersuchs }\end{array}$ & $\begin{array}{l}\text { Wieviel- } \\
\text { malige } \\
\text { Fallung }\end{array}$ & $\begin{array}{c}\text { Ange- } \\
\text { wandte } \\
\text { Substanz } \\
\text { umge- } \\
\text { rechnet }\end{array}$ & \multicolumn{2}{|c|}{ Gefunden } & $\begin{array}{c}\text { Darch- } \\
\text { schnitt } \\
\text { pC. }\end{array}$ \\
\hline I. a. & 2 & & 0,06533 & 6,98 & \\
\hline b. & & 0,9358 & 0,06452 & 6,89 & 6,93 \\
\hline c. & & 1 & 0,06492 & 6,93 & \\
\hline II. a. & 3 & 0,6320 & 0,04923 & 7,78 & \\
\hline b. & & 0,6255 & 0,04927 & 7,86 & 7,79 \\
\hline c. & & 1,2977 & 0,10032 & 7,73 & \\
\hline III. a. & 5 & 0,4986 & 0,03571 & 7,16 & \\
\hline b. & & 0,6943 & 0,05045 & 7,26 & 7,15 \\
\hline c. & & 1,2416 & 0,08725 & 7,03 & \\
\hline IV. a. & 7 & 1,9508 & 0,12221 & 6,26 & \\
\hline b. & & 0,5216 & 0,03300 & 6,32 & 6,25 \\
\hline c. & & 1,3668 & 0,08427 & 6,18 & \\
\hline
\end{tabular}

Es ergiebt sich also ein Natriumgehalt von 6 bis $8 \mathrm{pC}$., und zwar etwas verschieden je nach der Anzahl der Fällungen. Es folgt daraus erstens, dafs der Rohrzucker viel mehr Natrium und zwar doppelt soviel wie des an die Stärke, folglich nach den oben auseinandergesetzten Principien, dafs sein Moleculargewicht viel kleiner als dasjenige der Stärke ist. Ferner folgt aber auch aus den Zahlen, dafs das Rohrzuckernatrium bei Gegenwart von Wasser und Alkohol viel weniger zersetzlich *) ist als das Stärkenatrium, bei welchem sich der Natriumgehalt von $4,52 \mathrm{pC}$. auf $1,40 \mathrm{pC}$. je nach der Anzahl der Fällungen verminderte. Die Formel $\mathrm{C}_{12} \mathrm{H}_{21} \mathrm{O}_{11} \mathrm{Na}$ verlangt 6,32 pC. Natrium, hiergegen ist also $\mathrm{zu}$ viel gefunden, wenn man nicht unwahrscheinlicherweise annehmen will, dafs erst bei siebenmaligem Fällen die Verbindung rein wird. Es sind diese Zahlen etwas gröfser als die

*) Siehe auch J. Thomsen, Berichte der deutschen chemischen Gesellschaft $14,1650$. 
von Brendecke und von Soubeiran für die gleiche Verbindung gefundenen, denn $\mathrm{Br}$ endecke erhielt 6,08 pC. $\mathrm{Na}$ $\left(8,2\right.$ pC. $\left.\mathrm{Na}_{2} \mathrm{O}\right)$, Soubeiran 5,47 pC. $\mathrm{Na}\left(7,38\right.$ pC. $\left.\mathrm{Na}_{2} \mathrm{O}\right)$.

\section{Amylodextrin.}

Für den Zweck, die Moleculargröfse von Stärkederivaten zu bestimmen, schien dieser Körper besondere Vortheile zu bieten, denn augenscheinich steht das Amylodextrin zwischen Stärke und Maltose und es waren Resuitate zu erwarten zwischen den von uns mit der Stärke und den von Herzfeld mit der Maltose erhaltenen. Dabei schien das Amylodextrin durch seine krystallinische Form Garantie der Reinheit zu bieten *). Genau nach den Vorschriften von $\mathrm{N}$ ägeli haben wir deshalb das Amylodextrin auf folgende Weise darzustellen gesuclit. $1000 \mathrm{~g}$ Kartoffelstärke wurden mit 61 verdünnter Salzsäure $12 \mathrm{pC} . \mathrm{HCl}$ enthaltend, angesetzt und täglich durchgeschüttelt. Die dabei eintretenden Veränderungen, die Reactionen mit Jod u. s. w. entsprachen vollständig den Nägeli'schen Angaben. Nach 8 Wochen zeigten die Stärkekörner auf Zusatz von reinem Jod und Wasser eine vollständig gleichmäfsige gelbe Färbung und hielten wir deshalb die Umsetzung für beendigt.

Die Flüssigkeit wurde abgegossen, der Rückstand mit Wasser, schliefslich mit Alkohol so lange durch Decantiren ausgewaschen, bis sich in einer durch Kochen mit Wasser erhaltenen Lösung kein Chlor mehr nachweisen liefs. Die so gewonnene Masse bildete nach dem Trocknen ein schön weifses feines Pulver, welches unter dem Mikroskop die ursprünglichen Stärkeformen und im polarisirten Licht das bekannte schwarze Kreuz sehr schön zeigte.

*) W. Naggeli, Beitrige zur Kenntnife dèr Starkegruppe, Leipzig 1874; siehe such diese Analen $78,218$. 
Nach dem Lösen in kochendem Wasser und Filtriren zeigten sich, als wir nach $\mathrm{N}$ äg eli's Angabe die Lösung gefrieren liefsen, zwar nach dem Aufhauen äufserst kleine Sphärokrystalle, dieselben besafsen aber nie eine irgend nennenswerthe Einwirkung auf das polarisirte Licht. Wir haben dann das Ausfrieren unter den verschiedensten Bedingungen, ganz plötzlich bei $-15^{\circ}$, allmälig bei -2 bis $3^{0}$, aus concentrirter und aus verdünnter Lösung, mit Zusätzen von etwas Dextrin, Traubenzucker oder Glycerin vor sich gehen lassen, aber das Resultat blieb stets dasselbe. Endlich versuchten wir es mit der Drechsel'schen Alkoholdiffusion, indem wir $20 \mathrm{~g}$ rohes Amylodextrin in $50 \mathrm{ccm}$ Wasser gelöst erkalten liefsen, wodurch sich ziemlich viel ausschied, filtrirten und die abfiltrirte Lösung in einen Pergamentpapierdialysator brachten, der in einem gröfseren Gefäls mit Alkohol stand. Nach vollendeter Diffusion fanden sich in der ursprünglich wässrigen Lösung Sphärokrystalle, die bei einer 600 fachen Vergröfserung bei ihrer etwas ovalen Gestalt einen Querdurchmesser von 3 bis $4 \mathrm{~mm}$, einen Längsdurchmesser bis zu $5 \mathrm{~mm}$, umgerechnet also eine wirkliche Gröfse von 0,005 bis $0,006 \mathrm{~mm}$, resp. $0,0083 \mathrm{~mm}$ besalsen. Die meisten dieser Scheibchen zeigten deutliche, aber immerhin noch sehr schwache Polarisation. Wir versuchten das so gewonnene Product umzukrystallisiren, in der Hoffnung, die Kryställchen dadurch vergröfsern und damit die Polarisationsfähigkeit verstärken zu können, jedoch vergeblich, denn nach dem Ausfrieren war von einer Polarisation wieder nichts zu merken. Ungeachtet der nur theilweisen Uebereinstimmung mit $N$ ägeli's Beschreibung haben wir das Product in Arbeit genommen und zu dem Zweck sowohl mit dem Rohproduct, als auch mit verschiedenen daraus hergestellten anderen die Natriumverbindungen hergestellt, indem wir je $10 \mathrm{~g}$ mit $30 \mathrm{ccm}$ Wasser, im Mörser 
zerrieben, $1 \mathrm{~g}$ Natrium als Alkoholat hinzufügten, mit Alkohol fällten, wieder in Wasser lösten, zum zweitenmale fäititen $u_{\text {. }}$ s. w.

In der folgenden Tabelle bezeichnet :

1) Das Rohproduct.

2) Das Rohproduct in heifsem Wasser gelöst nnd duroh Ausfrioren das Amylodextrin wieder abgeschieden.

3) Robproduct in heifsem Wasser gelöst, mit Alkobol gefullt.

4) $200 \mathrm{~g}$ Rohproduct in $800 \mathrm{ccm}$ Wasser bei $70^{\circ} \mathrm{gelöst}$ und filtrirt. Hierbei schied sich wieder sehr viel aus (sieho IV). Das Filtrat wurde etwas eingedampft und mit Alkohol geftllt, wodurch wir circa $45 \mathrm{~g}$ Product erhiolten.

5) Filtrationsrückstand von 3 .

Bei der Analyse wurde die Substanz nur kurz über Schwefelsăure getrocknet, titrirt, in einer gleichzeitig in den Wassertrockenschrank gebrachten Probe die Feuchtigkeit bestimmt und hiernach die in der feuchten Probe gefundenen Natriumprocente auf Trockensubstanz umgerechnet*).

*) Vielleicht ist diefs Umrechnen nicht richtig, indem das bei $100^{\circ}$ ausgetriebene möglicherweise, wie z. B. in den Versuchen von Hönif und Bosenfeld (Berichto der deutschen chemischen Gesellschaft 10, 871; 12, 75), nicht hygroskopisches, sondern durch Zersetzung der Substanz herrorgegangenes Wasser ist.

Währeud Stärkenatrium sich beim Erhitzen auf $97^{\circ}$ kaum golblich fürbt, findet diels beim Rohrzuckernatrium in geringerem, boim Amylodextrinnatrium, Inulinnatrium und Dextrinnatrium, bhnlich wie in den Versuchen von Hönig und Rosenfeld, in hohem Mafse und ebenfalls, wenn auch langemer, auch schon bei gewöhnlicher Temperatur statt, und hiermit Hand in Hand geht eine Verminderung der Alkalitht bis endlich zur fast neutralen Reaction. Als das Amylodextrinnatrium ans dem Rohproduct (Berichte der deutschen chemischen Gesellschaft 18, 196) drei Stunden bei $97^{\circ}$ getrocknet war, hielt es nach dem Resultate der Titrirung 3,43 und $\mathbf{3 , 6 3} \mathrm{pC}$. Na, nach 8 stündigem Trocknen bei $100^{\circ}$ im Vacuum 1,20 und 1,24 pC. Na, nach 48stündigem Trocknen bei $97^{\circ}$ war die Alkalität kaum zu bostimmen. Es findet also in höherer Temperatur eine innere Saurebildung und Sättigung des vorhandeuen Alkali's statt, Hhnlich wie diefs von den Alkaliverbindungen der Zuckerarten bekannt ist, wolche sich leicht unter Bildung von Glucinsulure u. s. w., sowie besonders des Peligot'schen Baccharins zer- 
1. $0,9905 \mathrm{~g}$ Substanz gaben $0,03852 \mathrm{Na}=3,88 \mathrm{pC}$.

$0,8300 \mathrm{~g} n$ n $0,03205 \mathrm{Na}=3,86 \mathrm{pC}$.

$4,5065 \mathrm{~g} \mathrm{n} \quad \pi \quad 0,2743 \mathrm{H}_{2} \mathrm{O}=6,08$ pC. $\mathrm{H}_{2} \mathrm{O}$.

Also wasserfreie Substanz 4,12 pC. Na

8. $0,6761 \mathrm{~g}$ Substanz gaben $0,05032 \mathrm{Na}=7,44 \mathrm{pC}$.

0,8824 \& $n \quad 0,06343 \mathrm{Na}=7,19 \mathrm{pC}$.

$1,2762 \mathrm{~g} n$ " $0,0938 \mathrm{H}, \mathrm{O}=7,35 \mathrm{pC}$.

Hiernach anf wasserfreie Substanz umgerechnet 7,89 pC. Na.

3. $0,9465 \mathrm{~g}$ Substanz gaben $0,05615 \mathrm{Na}=5,93 \mathrm{pC}$.

$\begin{array}{llll}0,7447 \mathrm{~g} & n & n \quad 0,04319 \mathrm{Na}=5,80 \mathrm{pC} . \\ 0,6112 \mathrm{~g} & n & & 0,0184 \mathrm{H}_{8} \mathrm{O}=3,01 \mathrm{pC} .\end{array}$

Hiernach umgerechnet 6,04 pC. Na.

4. a. 0,6388 g Substanz gaben $0,03282 \mathrm{Na}=5,14 \mathrm{pC}$.

\begin{tabular}{|c|c|c|c|c|}
\hline 1,562 & $\mathrm{~g}$ & $n$ & ᄁ & $0,05434 \mathrm{Na}=5,14 \mathrm{pC}$. \\
\hline 0,5474 & g & $n$ & $n$ & $0,1012 \mathrm{H}_{2} \mathrm{O}=18,48 \mathrm{pC}$. \\
\hline 0,7508 & $\mathbf{g}$ & $n$ & $n$ & $0,04299 \mathrm{Na}=5,72 \mathrm{pC}$ \\
\hline 1,0220 & $\mathrm{~g}$ & $n$ & $n$ & $0,05787 \mathrm{Na}$ \\
\hline 0,5876 & $g$ & $n$ & $n$ & $0,0729 \mathrm{H}_{2} \mathrm{O}$ \\
\hline
\end{tabular}

Hiernach umgerechnet $6,48 \mathrm{pC}$. Na.

5. 0,6862 g Substanz gaben $0,04168 \mathrm{Na}=6,08 \mathrm{pC}$.

$1,0508 \mathrm{~g} n \quad 0,06397 \mathrm{Na}=6,09 \mathrm{pC}$.

$0,4217 \mathrm{~g} n \quad 0,0258 \mathrm{H}_{2} \mathrm{O}=6,11 \mathrm{pC}$.

Hiernach umgerechnet 6,48 pC. Na.

setzen, welche letzteres, wie besonders aus Scheibler's (Berichte der deutschen chemischen Gesellschaft 18,2212$)$ Untersuchungen hervorgeht, ebenfalls eine mit deutlich sauren Eigenschaften begabte Substanz ist. Sthrkenatrium dagegen braucht stets gleiche Mengen Săure zur Sättigung, sei es nar über Schwefelsaure getrocknet und auf Trockensubstanz bei $97^{\circ} \mathrm{um}$ gerechnet, sei es wirklich auf $97^{\circ}$ erhitzt geweson, wie unter anderem folgende Zahlen es beweisen :

a. Ueber Schwefelsäure getrocknot :

$1,2236 \mathrm{~g}$ Substanz gaben 0,03919 Na $=3,20 \mathrm{pC}$. $1,2992 \mathrm{~g} \quad$ " $0,04118 \mathrm{Na}=3,22 \mathrm{pC}$.

Wasserverlust bei $97^{\circ}$ :

$1,3968 \mathrm{~g}$ Substanz gaben 0,0937 $\mathrm{H}_{2} \mathrm{O}=6,7 \mathrm{pC}$.

Dansch umgerechnet 3,44 pC. Na.

b. Bei $97^{\circ}$ getrocknet :

0,3731 g Substanz gaben 0,01297 $\mathrm{Na}=5,48 \mathrm{pC}$.

$0,9600 \mathrm{~g} n \quad 0,03327 \mathrm{Na}=3,46 \mathrm{pC}$. 
Diese Resultate differiren von denen, welche mit der Stärke erhalten waren, sehr beträchtlich, denn sie bewegen sich zwischen 6,84 und 7,89 pC. Natrium, während Stärke im Durchschnitt 3,43 pC. Natrium aufnimmt. Nähere Uebereinstimmung mit letzterer Zahl zeigt nur die Natriumverbindung des ganz rohen Amylodextrins, letzteres möchte also der Stärke wohl noch ziemlich nahe stehen; die aus demselben durch Auflösen, Gefrieren u. s. w. gewonnenen Producte werden dagegen eine bedeutend einfachere Zusammenselzung haben, und die Zahlen deuten an, dafs besonders das durch Gefrieren gewonnene Amylodextrin (siehe I.) eine Zusammensetzung besitzt, welche mit derjonigen des Dextrins (siehe unten) fast übereinkommt.

\section{Dextrin.}

Aus Kartoffelstārke wurde nach O'Sulliva n's Vorschrift $\beta$-Dextrin erhalten, indem eine Mischung von $300 \mathrm{~g}$ Stärke und 51 kochendem Wasser nach dem Erkalten auf 65" mit einem Auszug von $40 \mathrm{~g} \mathrm{Malz}$ behandelt wurde. Nach 2 stündigem Erhitzen auf 60 bis $70^{\circ}$ trat keine rothe Reaction mit Jod mehr ein und es wurde aus der filtrirten und eingedampften Lösung nit Alkohol das Dextrin gefällt, mit neuem Alkohol bis zum Zähwerden behandelt und darauf zweimal in wenig Wasser wieder gelöst, mit Alkohol gefällt und mit Aether trocken gerieben. Ueber Schwefelsäure wurde es erst gummiartig, dann hart und brüchig. Ausbeute war $85 \mathrm{~g}$.

Die Darstellungsweise der Alkaliverbindungen war analog den früheren. Man erhält eine fadenziehende, seideglänzende Masse, die sich nur mit absolutem Alkohol vollständig trocken reiben läfst. Die Verbindungen sind sehr zersetzlich, denn schon bei 24 stúndigem Stehen über Schwefelsăure färbten 
sie sich gelblich, beim Trocknen bei $97^{\circ}$ rasch dunkelbraun.

\section{A. Natriumverbindung.}

$$
\begin{aligned}
& 0,8551 \text { \& Substanz gaben } 0,05805 \mathrm{Na}=6,79 \text { pC. } \\
& 1,5115 \mathrm{~g} n \quad \text { n } 0,10275 \mathrm{Na}=6,79 \mathrm{pC} \text {. } \\
& 1,1431 \mathrm{~g} n \text { n } 0,1335 \mathrm{H}_{\mathrm{g}} \mathrm{O}=11,68 \mathrm{pC} \text {. }
\end{aligned}
$$

Hiernach umgerechnet 7,70 pC. Na.

$$
\begin{array}{rlrl}
0,7345 & \text { gubstanz gaben } 0,04780 \mathrm{Na} & =6,51 \mathrm{pC} . \\
1,2643 \mathrm{~g} & n & n \quad 0,08251 \mathrm{Na} & =6,53 \mathrm{pC} . \\
1,0320 \mathrm{~g} & n & &
\end{array}
$$

Hiernach umgerechnet 7,68 pC. Na.

$$
\begin{array}{rl}
1,2560 \mathrm{~g} \text { Substanz gaben } 0,08531 \mathrm{Na} & =6,79 \mathrm{pC} . \\
0,9315 \mathrm{~g} n & \text { " } 0,06384 \mathrm{Na}=6,85 \mathrm{pC} . \\
0,9699 \mathrm{~g} n & n \quad 0,1192 \mathrm{H}_{\mathbf{4}} \mathrm{O}=12,30 \mathrm{pC} .
\end{array}
$$

Hiernach umgerechnet 7,77 pC. Na.

Von der bei $97^{\circ}$ getrockneten Masse machten wir eine Titration direct (a), eine nach vorherigem Veraschen *) der Substanz, um etwa gebildete organische Säure zu zerstören (b).

a. $0,3688 \mathrm{~g}$ Substan gaben $0,01085 \mathrm{Na}=2,92 \mathrm{pC}$.

b. $0,7342 \mathrm{~g}$ " $\quad 0,04854 \mathrm{Na}=6,60 \mathrm{pC}$.

Also ist bedentende invere Säurebildung eingetreten.

Kohlensäurebestimmung $\left.{ }^{* *}\right)$ :

$2,6000 \mathrm{~g}$ Substanz gaben $0,0160 \mathrm{CO}_{2}=0,61 \mathrm{pC}$.

$2,5846 \mathrm{~g} \quad$ " $0,0121 \mathrm{CO}_{2}=0,47 \mathrm{pC}$.

Im Burchschnitt auf wasserfreie Substanz umgerechnet 0,61 pC.

*) Das Veraschen mufs in einer verhaltnifsmalsig geräumigen offenen Platinschale vorgenommen werden, da die Verbindung beim Verkohlen sehr stark aufschwillt, wobei sich geringe Verlusto kaum vermeiden lassen.

*) Um die in der nur über Sohwefelsthre getrockneten Substanz zuräckgehaltenen Spuren Alkohol und Aether hierbei zuräckzuhalten, wurde vor die Absorptionsapparate ein Kugelapparat mit concentrirter Schwefelsaure eingeschaltet, dessen Inhalt sich braun färbte. 


\section{B. Dextrinkalium}

Auf analoge Weise abgestellt geben die Producte von vier Bereitungen folgende Zahlen :

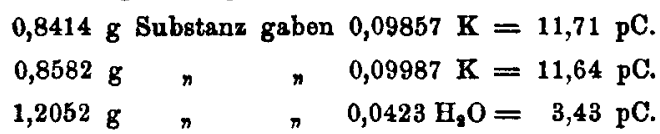

Hiernach umgerechnet 12,09 pC. $K$.

$1,7818 \mathrm{~g}$ Substanz gaben $0,21538 \mathrm{~K}=12,08$ pC.

$1,5534 \mathrm{~g}$ $, 0,18703 \mathrm{~K}=12,04 \mathrm{pC}$.

$1,2475 \mathrm{~g} n \quad 0,0964 \mathrm{H}_{2} \mathrm{O}=7,73 \mathrm{pC}$.

Hiernach umgerechnet 13,07 pC. K.

$1,3385 \mathrm{~g}$ Substanz gaben $0,14993 \mathrm{~K}=11,20 \mathrm{pC}$.

$1,2379 \mathrm{~g} n \quad 0,14119 \mathrm{~K}=11,40 \mathrm{pC}$.

$1,0304 \mathrm{~g} n \quad n \quad 0,1142 \mathrm{H}_{8} \mathrm{O}=10,57 \mathrm{pC}$.

Hiernach umgerechnet 12,63 pC. K.

1,0878 g Substanz gaben $0,12356 \mathrm{~K}=11,36$ pC.

$0,8464 \mathrm{~g} n \quad n \quad 0,09719 \mathrm{~K}=11,48 \mathrm{pC}$.

$0,9333 \mathrm{~g} n \rightarrow 0,0736 \mathrm{H}_{2} \mathrm{O}=7,89$ pC.

Hiernsch umgerechnet $12,53 \mathrm{pC}$. K.

Das Mittel aus den vier Bestimmungen beträgt 12,58 pC. K. Zur Kohlensäurebestimmung diente ein Gemenge der zur Kaliumbestimmung verwendeten Proben :

2,8920 g Substanz gaben $0,0267 \mathrm{CO}_{2}=0,92 \mathrm{pC}$.

$2,2814 \mathrm{~g} n \quad 0,0193 \mathrm{CO}_{2}=0,84 \mathrm{pC}$.

Auf wasserfreie Substanz umgerechnet 0,94 pC.

Schwer ist es, aus diesen Besultaten eine annehmbare Formel zu berechnen, am besten stimmt das Resultat der Alkalibestimmungen in der nur über Schwefelsäure getrockneten Substanz mit einer 12 At. Kohlenstoff haltenden Formel.

\begin{tabular}{lccc}
\multicolumn{2}{c}{$\begin{array}{l}\text { Berechnet nach } \\
\mathrm{C}_{19} \mathrm{H}_{10} \mathrm{O}_{10} \mathrm{M}\end{array}$} & $\begin{array}{l}\text { ïber Schwe- } \\
\text { felsäure } \\
\text { getrocknet }\end{array}$ & $\begin{array}{c}\text { auf Trocken- } \\
\text { substanz } \\
\text { berechnet }\end{array}$ \\
Natriumverbindung & 6,65 & 6,71 & 7,74 \\
Kaliumverbindung & 10,80 & 11,61 & $12,58$.
\end{tabular}


Möglicherweise ist etwas Dextrose, wolche als Natriumverbindung $11,4 \mathrm{pC}$. Na, als Kaliumverbindung 17,9 pC. K enthalt, beigemengt gewesen.

Auf jeden Fall aber zeigt siçh der grofse Unterschied im Gehalt der Dextrin- und der Stärkeverbindungen einerseits und der Dextrin- und der Dextroseverbindungen andererseits; es folgt daraus, dafs die einfache Formel $\mathrm{C}_{6} \mathrm{H}_{10} \mathrm{O}_{5}$ für das Dextrin nicht anwendbar ist, und einstweilen behält man wohl am besten die Formel $\mathrm{C}_{12} \mathrm{H}_{20} \mathrm{O}_{10}$ oder $\mathrm{C}_{12} \mathrm{H}_{22} \mathrm{O}_{11}$, welche auch von anderen Seiten aufgestell ist, als die wahrscheinlichste bei.

\section{Inulin.}

Das käufliche Inulin löste sich in Wasser mit etwas gelblicher Farbe und wurde vor dem Gebrauch durch einmaliges Ausfrieren und Fällen mit Alkohol gereinigt. Wie Dragendorf $f^{*}$ ) anführt, sind schon von Braconnot, Parnell, Crookwit, Mulder, Berzelius, Sachs u. A. Beobachtungen über die Einwirkung von Alkalien auf Inulin gemacht. Er selbst hat die betreffenden Verbindungen dargestellt, aber sehr verschiedene Resultate erhalten. So fand er 10,95 bis $28,4 \mathrm{pC} . \mathrm{K}_{\mathbf{2}} \mathrm{O}$ im Inulinkalium, und in einer Inulinnatriumprobe 20,89 pC. $\mathrm{Na}_{2} \mathrm{O}$. Die hohen Zahlen sind wohl dadurch erhalten, dafs ein Ueberschufs von Alkali, wie bei den Stärkeverbindungen gezeigt ist, nur durch zweimaliges Fällen und sorgfältiges Auswaschen beseitigt werden kann, während Dragendorff den durch Alkohol bewirkten Niederschlag direct analysirte. Uebrigens mifst $D$ ragendorff den von ihm aufgestellten Formeln $\left(\mathrm{C}_{6} \mathrm{H}_{9} \mathrm{NaO}_{3}\right)_{2}+\mathrm{Na}_{2} \mathrm{O}+2 \mathrm{H}_{2} \mathrm{O}$ oder $\mathrm{C}_{6} \mathrm{H}_{9} \mathrm{NaO}_{5}+\mathrm{H}_{2} \mathrm{O}$ selbst keinen grofsen Werth bei.

\section{A. Inulinnatrium.}

Bei der Darstellung des Inulinnatriums mufs man, um sich

*) Drageudorff, Materialien, B. 121. 
vor Verlusten za schützen, mit möglichst geringen Mengen Wasser operiren. Die Verbindung ist weniger zersetzlich als Dextrinnatrium, denn sie blieb beim Stehen über Schwefelsäure völlig weifs *); beim Trocknen bei $97^{\circ}$ läfst sie jedoch eine bemerkenswerthe innere Sätligung erkennen, was aus nachfolgenden Zahlen hervorgeht.

Bei $97^{\circ}$ getrocknet und direct titrirt gaben

0,9727 g Substanz $0,04281 \mathrm{Na}=4,40 \mathrm{pC}$.,

nach vorherigem Veraschen titrirt gaben dagegen

0,3482 g Substanz $0,02333 \mathrm{Na}=6,69 \mathrm{pC}$.

Die weiteren Alkalibestimmungen wurden daher wieder in der oben angegebenen Weise ausgeführt.

0,6287 g Substanz gaben $0,04033 \mathrm{Na}=6,41 \mathrm{pC}$.

$0,8595 \mathrm{~g} n \quad 0,05524 \mathrm{Na}=6,42 \mathrm{pC}$.

$0,7986 \mathrm{~g} \rightarrow \quad 0,0468 \mathrm{H}_{2} \mathrm{O}=5,86 \mathrm{pC}$.

Hiernach umgerechnet 6,80 pC. Na.

0,5258 g Substanz gaben $0,03219 \mathrm{Na}=6,12 \mathrm{pC}$.

$0,9869 \mathrm{~g}, n \quad 0,05976 \mathrm{Na}=6,06 \mathrm{pC}$.

$1,1082 \mathrm{~g} n$ n $0,1130 \mathrm{H}_{2} \mathrm{O}=10,21 \mathrm{pC}$.

Hiernach umgerechnet 6,75 pC. Na.

\section{Rohlensäurebestimmung :}

2,9721 g Bubstanz gaben $0,0049 \mathrm{CO}_{\mathrm{z}}=0,17 \mathrm{pC}$.

$2,6407 \mathrm{~g} n \quad 0,0041 \mathrm{CO}_{2}=0,15 \mathrm{pC}$.

Zur Verbrenuung wurde das Product bei $97^{\circ}$ getrocknet.

I. $0,2320 \mathrm{~g}$ Substanz gaben $0,3429 \mathrm{CO}_{2}$ und $0,1288 \mathrm{H}_{2} \mathrm{O}$.

II. $0,5703 \mathrm{~g} n \quad 0,8472 n$ " $\quad 0,3089$ "

III. $0,4308 \mathrm{~g} n \quad 0,02535 \mathrm{Na}$ und $10,21 \mathrm{pC}$. Wasserverlust.

\begin{tabular}{cccccr} 
& \multicolumn{2}{c}{ Berechnet für } & & \multicolumn{2}{c}{ Gefunden } \\
\cline { 3 - 4 } & $\mathrm{C}_{12} \mathrm{H}_{10} \mathrm{O}_{10} \mathrm{Na}$ & $\mathrm{C}_{1,} \mathrm{H}_{91} \mathrm{O}_{11} \mathrm{Na}$ & & I. & II. \\
$\mathrm{H}$ & 41,62 & 39,56 & & 40,45 & 40,51 \\
$\mathrm{Na}$ & 5,49 & 5,77 & & 6,16 & 6,02 \\
& 6,65 & 6,32 & & \multicolumn{2}{c}{$6,67}$.
\end{tabular}

*) Jetzt, d. h. nach circa 6 Monaten, ist sie in einem Glase aufbewahrt braun und weich geworden. 
B. Inulinkalium.

Zar Bereitung diente (a) Inulin von derselben Portion, welche zum Inulinnatrium angewandt war, doch hatte sich diese etwas durch Feuchtigkeit zersetzt und war deshalb vorher noch einmal in Wasser gelöst und mit Alkohol gefällt worden.

Ferner wurde (b) Inulin, welches aus früh geernteten Knollen von Helianthus tuberosus gewonnen war, hierzu verwendet. Die erhaltenen Producte waren ziemlich stark gefärbt.

Die Probe (a) gab folgende Resultate :

1. $0,6364 \mathrm{~g}$ Substanz gaben $0,07435 \mathrm{~K}=11,68 \mathrm{pC}$.

$$
\begin{array}{llll}
0,9224 \mathrm{~g} & \text { n } & n & 0,10746 \mathrm{~K}=11,65 \mathrm{pC} . \\
0,4322 \mathrm{~g} & n & n & 0,0308 \mathrm{H}_{8} \mathrm{O}=7,13 \mathrm{pC} .
\end{array}
$$

Hiernach umgerechnet 12,56 pC. $\mathrm{K}$.

2. $0,6945 \mathrm{~g}$ Substanz gaben $008109 \mathrm{~K}=11,61 \mathrm{pC}$.

$$
\begin{array}{llll}
1,5663 \mathrm{~g} & \text { " } & n & 0,18411 \mathrm{~K}=11,75 \mathrm{pC} . \\
0,3760 \mathrm{~g} & \text { n } & \text { " } & 0,0263 \mathrm{H}_{2} \mathrm{O}=7,0 \mathrm{pC} .
\end{array}
$$

Hiernach umgerechnet $12,56 \mathrm{pC}$. K.

\section{Kohlensäurebestimmung :}

2,0827 g Substanz gaben 0,0131 $\mathrm{CO}_{2}=0,63 \mathrm{pC}$.

$2,1415 \mathrm{~g} n \quad 0,0125 \mathrm{CO}_{2}=0,58 \mathrm{pC}$.

Auf wasserfreie Substane umgerechnet $0,64 \mathrm{pC}$.

Die Probe (b) dagegen :

$$
\begin{aligned}
& 0,8443 \mathrm{~g} \text { Substanz gaben } 0,10700 \mathrm{~K}=12,67 \mathrm{pC} \text {. } \\
& 0,9123 \mathrm{~g} \quad \pi \quad \text { n } 0,11666 \mathrm{~K}=12,78 \mathrm{pC} \text {. } \\
& 1,3159 \mathrm{~g} n \quad \text { n } 0,0988 \mathrm{H}_{2} \mathrm{O}=7,5 \text { pC. }
\end{aligned}
$$

Hiernach umgerechnet 13,75 pC. K.

Diese Proben enthielten also auffällig viel Kalium im Vergleich mit den Resultaten der Natriumverbindung, und es ist möglich, dafs das angewandte Material die Schuld daran trägt; denn sowohl in Inulin aus Helianthus, als auch in etwas verändertem Inulin kann mehr oder weniger des ersten Umwandlungsproducts desselben, des Lävulins, vorhanden sein, und speciell in dem Producte (b) ist die Gegenwart des letz- 
teren fast unausbleiblich, da das Làvulin in grofsen Mengen in der genannten Knolle stets vorhanden ist, während das Inulin überhaupt nur in ziemlich früher Vegetationszeit darin sich findet und bald durch Lïvulin ersetzt wird. Auf Grund der Natriumverbindung, welche mit unverändertem Inulin hergestellt war, glauben wir jedoch dem Inulin, unter den mehrfach erwähnten allgemeinen Reserven, die Formel $\mathrm{C}_{18} \mathrm{H}_{80} \mathrm{O}_{10}$ (eventuell mit etwas Wasser) zuschreiben zu können, während sich aus den Kaliumbestimmungen eine noch kleinere Formel ergeben würde.

Auf jeden Fall zeigt sich eine sehr auffallende Differenz zwischen den Alkaliverbindungen des Inulins und der Stärke, und aus den Eingangs dieser Arbeit entwickelten Gründen zwischen den Moleculargröfsen der genannten beiden Kohlehydrate. Man darf deshalb nicht Inulin und Stärke, wie diefs vielfach geschehen ist, in Parallele stellen. Man mufs sie vielmehr als Körper verschiedener Ordnung auseinanderhalten *), und diefs Resultat steht im Einklange mit dem aus sonstigen Beobachtungen und Umsetzungen der genannten beiden Körper zu ziehenden Schlusse, wonach das Inulin dem daraus sich bildenden Zucker, der Lävulose, sehr viel näher steht, als die Stärke der sich schliefslich aus ihr bildenden Dextrose.

\section{Rückblick.}

Die Resultate von vorliegender Arbeit lassen sich in Folgendem kurz zusammenfassen :

1) Ein Urtheil über die Moleculargröfse der Körper der Stärkereihe läfst sich mit Hülfe der Alkaliverbindungen dieser Kohlehydrale gewinnen, doch sind die von uns gefundenen Formeln aus den oben dargelegten Gründen vielleicht nicht völlig genau, vielleicht auch nur als Minimalgröfsen zu betrachten.

*) Vgl Dieck und Tollens, dieso Amselen 198, 246. 
2) Der Stärke kommt unter obigen Reserven die Formel $\mathrm{C}_{24} \mathrm{H}_{40} \mathrm{O}_{20}$ oder $\mathrm{C}_{24} \mathrm{H}_{48} \mathrm{O}_{21} \mathrm{zu}$, welche vier alte Stärkegruppen $\mathrm{C}_{6} \mathrm{H}_{10} \mathrm{O}_{5}$ umfarst.

3) Die Formel des Rohrzuckers $\mathrm{C}_{12} \mathrm{H}_{22} \mathrm{O}_{11}$ wird durch die auch von uns gefundene Zusammensetzung seiner Natriumverbindung bestätigt.

4) Das Inulin besitzt eine Formel mit 12 Atomen Koblenstoff, d. h. $\mathrm{C}_{12} \mathrm{H}_{20} \mathrm{O}_{10}$ oder $\mathrm{C}_{12} \mathrm{H}_{29} \mathrm{O}_{11}$, und eine Parallelstellung derselben mil der Stärke ist daher unhaltbar.

5) Dextrin hat weniger stimmende Resultate ergeben, doch folgt aus den erhaltenen Zahlen, dafs die Moleculargröfse des Dextrins viel geringer ist als diejenige der Stärke und sich mehr derjenigen der Zuckerarten und des Inulins nähert.

6) Amylodextrinnatrium aus rohem Amylodextrin hat Zahlen ergeben, welche sich denen der entsprechenden Stärkeverbindungen nähern. Durch Ausfrieren, Ausfällen und andere Manipulationer gewonnene Amylodextrine haben dagegen Zahlen geliefert, welche mehr oder weniger mit denen des Dextrins, des Inulins, des Rohrzuckers übereinstimmen.

Göt ting e n, agricultur-chemisches Laboratorium, August 1881. 